\title{
SOX10 directly modulates ERBB3 transcription via an intronic neural crest enhancer
}

\author{
Megana K Prasad ${ }^{1 \dagger}$, Xylena Reed ${ }^{1}$, David U Gorkin ${ }^{1}$, Julia C Cronin², Anthony R McAdow ${ }^{3}$, Kristopher Chain ${ }^{3}$, \\ Chani J Hodonsky ${ }^{4}$, Erin A Jones ${ }^{6}$, John Svaren ${ }^{7}$, Anthony Antonellis ${ }^{4,5}$, Stephen L Johnson ${ }^{3}$, Stacie K Loftus ${ }^{2}$, \\ William J Pavan ${ }^{2}$ and Andrew S McCallion ${ }^{1,8^{*}}$
}

\begin{abstract}
Background: The ERBB3 gene is essential for the proper development of the neural crest (NC) and its derivative populations such as Schwann cells. As with all cell fate decisions, transcriptional regulatory control plays a significant role in the progressive restriction and specification of NC derived lineages during development. However, little is known about the sequences mediating transcriptional regulation of ERBB3 or the factors that bind them.

Results: In this study we identified three transcriptional enhancers at the ERBB3 locus and evaluated their regulatory potential in vitro in NC-derived cell types and in vivo in transgenic zebrafish. One enhancer, termed ERBB3_MCS6, which lies within the first intron of ERBB3, directs the highest reporter expression in vitro and also demonstrates epigenetic marks consistent with enhancer activity. We identify a consensus SOX10 binding site within ERBB3_MCS6 and demonstrate, in vitro, its necessity and sufficiency for the activity of this enhancer. Additionally, we demonstrate that transcription from the endogenous Erbb3 locus is dependent on Sox10. Further we demonstrate in vitro that Sox10 physically interacts with that ERBB3_MCS6. Consistent with its in vitro activity, we also show that ERBB3_MCS6 drives reporter expression in NC cells and a subset of its derivative lineages in vivo in zebrafish in a manner consistent with erbb3b expression. We also demonstrate, using morpholino analysis, that Sox10 is necessary for ERBB3_MCS6 expression in vivo in zebrafish.
\end{abstract}

Conclusions: Taken collectively, our data suggest that ERBB3 may be directly regulated by SOX10, and that this control may in part be facilitated by ERBB3_MCS6.

\section{Background}

The neural crest $(\mathrm{NC})$ is a transient, multipotent and migratory population of cells present in early vertebrate development. NC cells arise from the lateral folds of the neural plate at neurulation and give rise to a multitude of cell types including pigment cells, neurons and glia of the peripheral nervous system, craniofacial skeleton and cartilage, and adrenal medullary cells [1]. Defects in NC development underlie several human diseases such as Waardenburg syndrome, Hirschsrpung disease and DiGeorge syndrome, which present a spectrum of phenotypes including craniofacial defects, ocular,

\footnotetext{
* Correspondence: andy@jhmi.edu

+ Contributed equally

'McKusick-Nathans Institute of Genetic Medicine, The Johns Hopkins

University School of Medicine, Baltimore, Maryland 21205, USA

Full list of author information is available at the end of the article
}

pigmentary and otic defects, enteric hypoganglionosis, and cardiac malformations [2,3]. Despite significant recent progress in the identification of key signaling pathways and transcription factors involved in NC induction, the hierarchical relationships between these pathways and factors are not well understood.

One critical gene in this network is Erbb3, a receptor tyrosine kinase that belongs to the epidermal growth factor (EGF) receptor family. Other members of the family include receptors such as Egfr1, Erbb2 and Erbb4 [4]. The structure of Erbb3 includes an extracellular domain, which interacts with ligands Neuregulin1 and Neuregulin2, and a cytoplasmic domain. Unlike other members of the EGF receptor family, the cytoplasmic domain of Erbb3 lacks tyrosine kinase activity [5,6]. Erbb3 is therefore thought to heterodimerize with Erbb2, which lacks a cognate receptor but possesses

\section{Biomed Central}


tyrosine kinase activity, in order to activate downstream pathways $[7,8]$. Upon activation, Erbb3 triggers several downstream pathways such as PI3K, MAPK, protein kinase C, Jak-STAT and PLC $\gamma$ [4].

Erbb3 plays important roles in the development of the $\mathrm{NC}$ and its derivative tissues. Consistent with these observations, expression analyses in mouse and zebrafish have detected Erbb3/erbb3b transcripts in pre-migratory and migratory $\mathrm{NC}$, and various $\mathrm{NC}$ derivatives including, cranial ganglia, posterior lateral line ganglia and Schwann cells [9-14]. Expression has also been observed in non-NC derived populations including the brain, olfactory lobes and myotome $[10,14]$. Furthermore, mice harbouring targeted mutant alleles of Erbb3 exhibit defects in the formation of $\mathrm{NC}$ and its derivatives. Homozygous null mice display a dramatic reduction in numbers of Schwann cells, hypoplastic cardiac cushion mesenchyme and cardiac valves, defects in cranial ganglia formation and cerebellar hypoplasia [9,13]. Erbb3 deficient mice also demonstrate a lack of chromaffin cells in the adrenal medulla [9]. Furthermore, targeted deletions of Nrg1 and Erbb2, a ligand and a binding partner of Erbb3 respectively, result in NC cell migration defects [15]. More recently, several in vitro and zebrafish studies have revealed a potential role for Erbb3 in melanocyte development and in melanoma [16-18]. Consistent with these data, Erbb3 has been implicated in other neoplasias including breast cancer and lung cancer [19]. However, what factors act upstream of $E r b b 3$ to direct its expression and function in the variety of cell types derived from NC cells is not well understood. Even less well understood is the genomic sequence basis of the transcriptional regulatory control that facilitates the cell fate decisions and homeostatic maintenance of these cells during development

One factor proposed to regulate Erbb3 is Sox10, a member of the SoxE family of transcription factors [20]. Sox10 is indispensible for proper neural crest development and loss of Sox10 leads to reductions of specific NC derivatives including peripheral neurons and melanocytes $[21,22]$. Also, SOX10 mutations have been discovered in several patients with Waardenburg syndrome and syndromic Hirschsprung disease [23]. However, data generated to date linking Sox 10 and Erbb3 have been largely correlative. Both Sox10 and Erbb3 share overlapping expressions patterns and deletions of Sox10 and Erbb3 affect overlapping cell populations such as Schwann cells, cranial ganglia and sympathetic neurons. In mice where the endogenous Sox10 locus has been replaced by a LacZ cassette, Erbb3 expression is initiated in premigratory $\mathrm{NC}$ but is lost once the cells begin migrating [10], suggesting Erbb3 regulation by Sox10. Furthermore Erbb3 transcript levels increase upon overexpression of Sox10 in Neuro2A neuroblastoma cells
[10]. However, it is not known if this regulation is direct or indirect because the regulatory topography of Erbb3 has not yet been uncovered, and nor have any Sox 10 responsive elements been demonstrated at the Erbb3 locus.

In this study, we address the transcriptional regulation of $E R B B 3$ during neural crest development. We use sequence constraint as a metric predictive of function to identify putative regulatory elements at the human $E R B B 3$ locus. We test the regulatory potential of these elements in vitro in two cell lines representative of neural crest derived tissues. We also test each element for epigenetic marks consistent with enhancer activity. We verify enhancer activity of each element in vivo using zebrafish transgenesis, identifying three transcriptional enhancers of $E R B B 3$. One intronic enhancer termed ERBB3_MCS6 displays the strongest enhancer activity in vitro and high enrichment for H3K4me1. We demonstrate that this enhancer is responsive to and dependent on Sox10 for its regulatory behaviour. Similarly, we demonstrate that Sox10 is necessary and sufficient for activation of the endogenous Erbb3. Furthermore, we also demonstrate that ERBB3_MCS6 directs expression in $\mathrm{NC}$ cells and their derivative lineages in vivo in zebrafish, and that the requirement for Sox 10 for its regulation is maintained in vivo as well. Therefore, we conclude that SOX10 likely contributes to the transcriptional modulation of $E R B B 3$ acting at least in part directly via ERBB3_MCS6.

\section{Results}

Multi-species conserved sequences (MCS) at the ERBB3 locus direct reporter expression in Erbb3 expressing cell lines and show epigenetic marks consistent with enhancer activity

As in previous studies, we used sequence constraint to identify putative regulatory enhancers of ERBB3 [24,25]. Briefly, we identified evolutionarily conserved non-coding sequences at the human ERBB3 locus using Phastcons (http://genome.ucsc.edu/) [26], selecting eight sequences for functional analyses (LOD 30-196; Methods; Figure 1A). We amplified and cloned each of the sequences upstream of an E1B minimal promoter driving a luciferase reporter. We tested the regulatory potential of each of the sequences to drive transcription via luciferase assays in two NC derived cell lines, cultured mouse melanocytes (melan-a) and cultured rat Schwann cells (S16), both of which express Erbb3 (Figure $1 \mathrm{~B}, \mathrm{C})$. Of the eight MCS elements amplified and tested, five (ERBB3_MCS2, MCS3, MCS4, MCS6 and MCS7) directed luciferase reporter expression at levels significantly higher than the promoter only luciferase construct in both cell lines $(\mathrm{p} \leq 0.003)$ (Figure 1B, C). Furthermore, ERBB3_MCS6 showed the greatest 


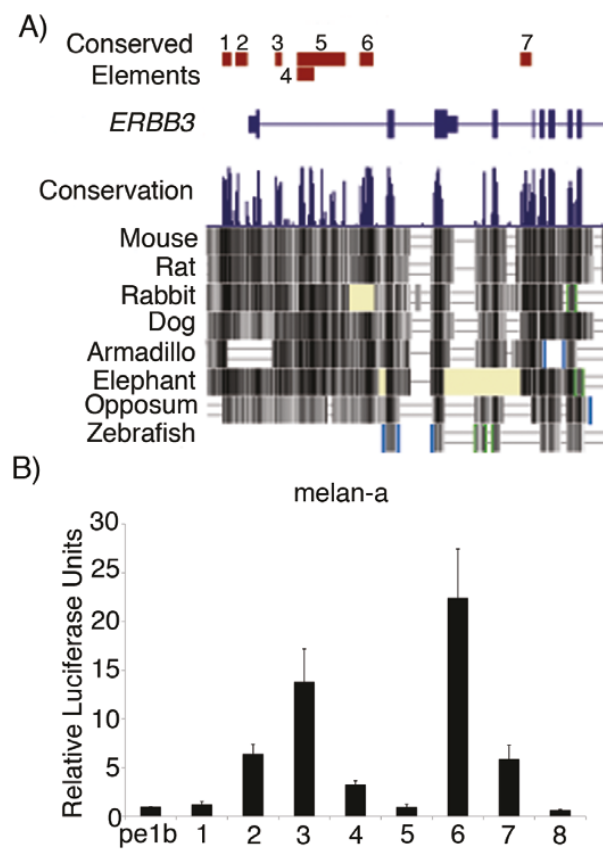

D)

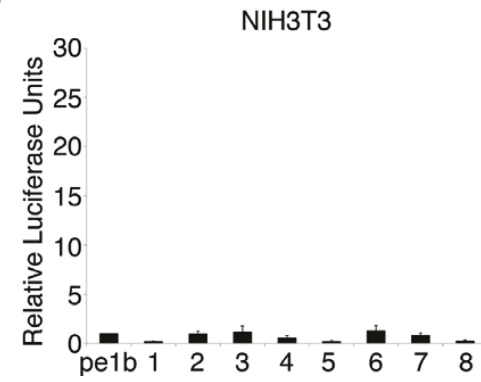

C)

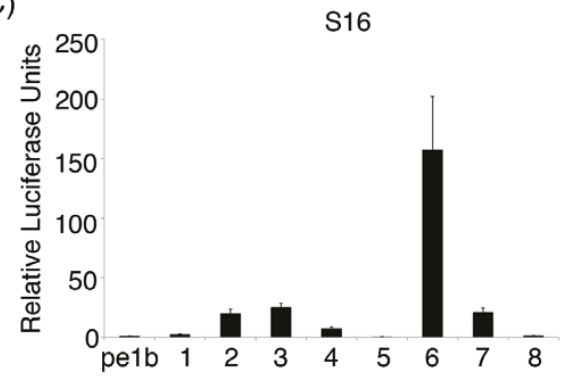

E)

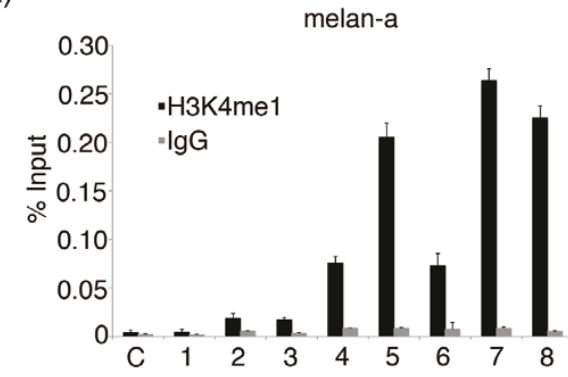

Figure 1 Multi-species conserved sequences at the ERBB3 drive reporter activity in neural crest derived lines and exhibit epigenetic marks consistent with enhancer activity. (A) Schematic representation of the eight multi-species conserved sequences at the human ERBB3 locus. (B) Luciferase activity of ERBB3_MCS1-8 in melan-a cells. (C) Luciferase activity of ERBB3_MCS1-8 in S16 cells. (D) Luciferase activity of ERBB3_MCS1-8 in NIH3T3 cells. All luciferase values are normalized to internal renilla control and are shown as fold-change in reporter activity as compared to the promoter only construct (pe1b) with standard deviation. (E) Real-time PCR results of Chromatin Immunoprecipitation. Black bar indicates enrichment upon H3K4me1 ChIP whereas grey bar indicates enrichment with non-specific IgG. Error bars indicate standard deviation of technical replicates in real-time PCR. Elements MCS1-8 represent the mouse orthologs of ERBB3_MCS elements. C refers to a region 3 Mb upstream of Erbb3, which was used as a negative control.

increase in luciferase activity- approximately 23-fold greater than the promoter only construct $(\mathrm{p}=0.002)$ in melan-a cells and approximately 150 -fold greater than the promoter only construct in S16 cells. Interestingly, although ERBB3_MCS5 overlaps ERBB3_MCS4, it demonstrates lower luciferase activity than MCS4 in melan-a cells. This suggests that there may be transcriptional repressor elements in ERBB3_MCS5 that are causing it to drive lower reporter expression in the luciferase assay. When the eight ERBB3_MCS elements were tested in a cell line that does not express Erbb3 (NIH3T3), none directed higher luciferase activity than the promoter only construct (Figure 1D). Therefore, this suggests that ERBB3_MCS 2, MCS3, MCS4, MCS6 and
MCS7 are potentially enhancers of ERBB3 expression in the NC.

In order to determine whether these MCS elements were indeed enhancers within their genomic context, we assayed the presence of an epigenetic mark previously shown to be predictive of enhancer function. Since H3K4 monomethylation (H3K4me1) has been shown to be enriched at transcriptional enhancers, we used chromatin immunoprecipitation (ChIP) to determine whether H3K4me1 marks were enriched at the mouse orthologs of ERBB3_MCS elements within the genomic context of melan-a cells [27]. We used an antibody against H3K4me1 and an isotype control IgG to immunoprecipitate sheared crosslinked chromatin from these 
cells. We analyzed immunoprecipitation using real-time PCR and calculated enrichment using the percent input method (Figure 1E). ERBB3_MCS1, MCS2 and MCS3 showed consistently low levels of enrichment upon immunoprecipitation with the H3K4me1 antibody as compared to the IgG control samples, whereas ERBB3_MCS4, MCS5, MCS6, MCS7 and MCS8 showed consistently high levels of enrichment upon immunoprecipitation as compared to the IgG samples. A control sequence $3 \mathrm{Mb}$ upstream of ERBB3 was not enriched in H3K4me1 samples as compared to IgG samples. ERBB3_MCS5, MCS7 and MCS8 showed the highest levels of enrichment as compared to the IgG samples. This contrasts with the low luciferase activity driven by these sequences in vitro, consistent with reports suggesting that H3K4me1 is not sufficient to distinguish between functionally active and inactive enhancers $[28,29]$. Elements ERBB3_MCS2, MCS3, MCS4 and MCS6 however did show increased luciferase activity as well as H3K4me1 marks, suggesting that these sequences may be functional endogenous enhancers of $E R B B 3$ in NC-derived cell types.

\section{ERBB3_MCS6 harbours putative transcription factor binding sites for key neural crest transcription factors} Since ERBB3_MCS6 demonstrated the highest luciferase activity in vitro and showed enrichment for H3K4me1 consistent with a role for ERBB3_MCS6 as an enhancer of $E R B B 3$, we focused our subsequent analyses on this element. To determine what pathways or transcription factors may be acting upon this element and hence potentially modulating Erbb3 expression, we queried three publicly available transcription factor identification databases (JASPAR, MATCH and TRANSFAC) to identify putative transcription factor binding sites (TFBSs) within this sequence. To refine our search further we prioritized sites that were found by two or more TF databases, selecting those that corresponded to transcription factors with known roles in NC development (SOXE and AP2). We identified two SOXE sites (SOXE1 and SOXE-2) and one AP2 site (Additional file 1, Figure S1). Members of the SOXE family, SOX8, SOX9 and SOX10, bind to the SOXE consensus site 5'AACAAT-3'. The identification of SOXE family (SOX8, SOX9 and SOX10) motifs is consistent with the predicted role for Sox10 in Erbb3 modulation. SOXE proteins typically bind the consensus site 5'AACAAT-3'. Both SOXE-1 and SOXE-2 contain the 5'-ACAAT-3' core sequence but differ from the consensus in that the $5^{\prime}$-most adenine is replaced by a cytosine. AP2 is a retinoic acid inducible transcription factor that binds to the consensus sequence 5'-CCCCAGGC-3' [30,31]. It is expressed in the $\mathrm{NC}$ and its derivatives including cranial and sensory ganglia and facial mesenchyme and has a known role in the regulation of $E R B B 3$ expression [32-36]. The ERBB3 promoter harbours potential AP2 binding sites, causing speculation of the role of AP2 as an $E R B B 3$ regulator [37].

\section{SOXE2 is required for enhancer activity of ERBB3_MCS6}

We set out to determine the importance of the SOXE and AP2 transcription factor families in modulating the activity of ERBB3_MCS6 by mutating predicted binding sites in the context of a pe1b promoter driving a luciferase reporter. We then compared the luciferase activity of each mutant construct to that of the wild-type ERBB3_MCS6 sequence in the melan-a cell line, which expresses Sox10, Erbb3 and Ap2 (Figure 2A). Mutation of the SOXE2 but not the SOXE1 motif significantly reduced enhancer activity of ERBB3_MCS6 (Figure 2A, $\mathrm{p}=0.002$ for SOXE2). This observation is consistent with a suggested role for Sox10 in regulating Erbb3 in $\mathrm{NC}$-derived populations [10]. However, since mutation of the SOXE-2 site does not completely abrogate $E R B B 3$ 3CS6, this suggests that additional factors may also be responsible for the activity of ERBB3_MCS6. By contrast, mutation of the AP2 site does not significantly reduce luciferase activity of ERBB3_MCS6 across replicate experiments. However, we cannot exclude the presence of a non-canonical AP2 site that may be required for ERBB3_MCS6 activity independent of or in addition to this site.

\section{Sox10 is necessary for ERBB3_MCS6 enhancer activity and Erbb3 expression}

Since mutation of the SOXE-2 binding site caused a significant reduction in ERBB3_MCS6 enhancer activity, we asked whether Sox10 may be acting through ERBB3_MCS6 to regulate Erbb3 in NC populations. We used siRNA (SMARTpool, Dharmacon) to transiently knock down Sox10 gene product in melan-a cells and assayed the effect of the knockdown on ERBB3_MCS6 activity via luciferase assays, as before. Consistent with significantly reduced ERBB3_MCS6 activity (Figure 2B; $\mathrm{p}=0.05)$. Sox10 knockdown was confirmed via Western blot as shown in Figure $2 \mathrm{C}$. We also determined the effect of Sox10 knockdown on endogenous Erbb3 protein levels in melan-a cells. As shown in figure 2D, knockdown of Sox 10 causes a decrease of endogenous Erbb3 protein levels. Similarly, we interfered with Sox10 protein function in S16 cells using a previously characterized construct expressing a dominant negative mutant form of Sox10 (E189X) under the control of a CMV promoter [38]. The E189X mutant protein has an intact DNA binding domain but lacks a transactivation domain. Upon transfection of S16 cells with this mutant cDNA of Sox10, there was a significant decrease in luciferase activity of ERBB3_MCS6 (Figure 2E). Taken 

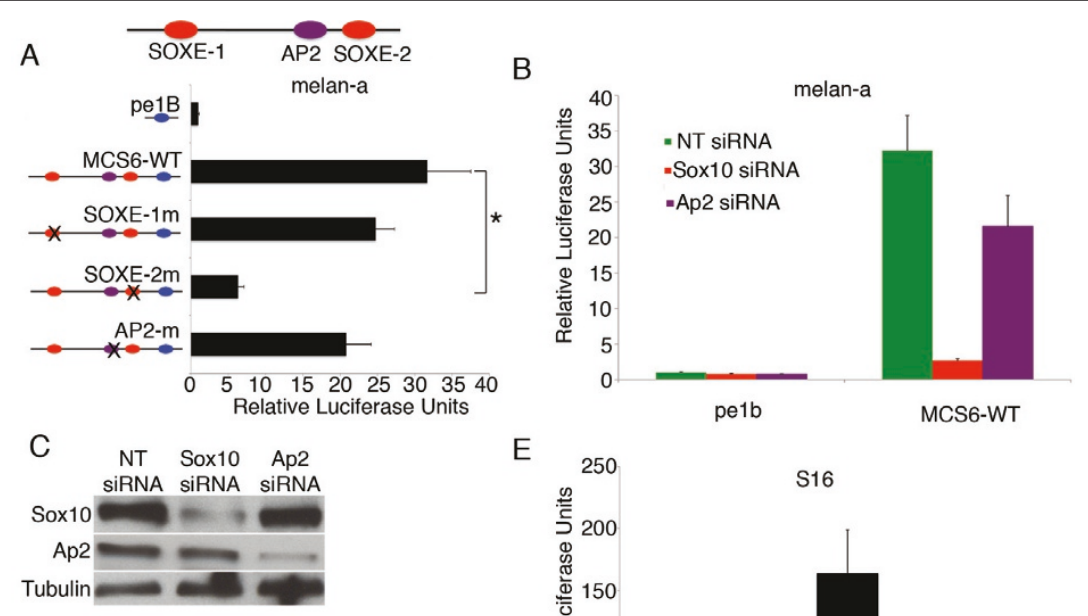

E

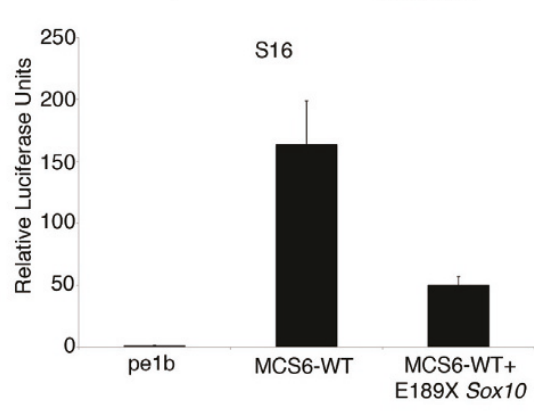

$\mathrm{F}$
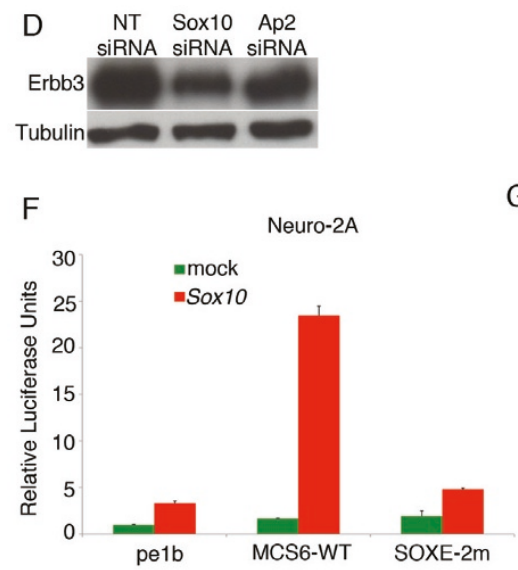

$\mathrm{H}$

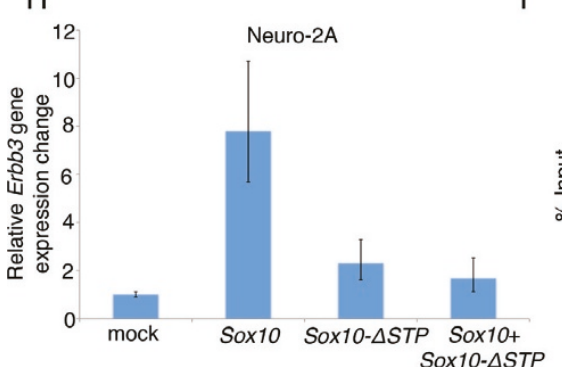

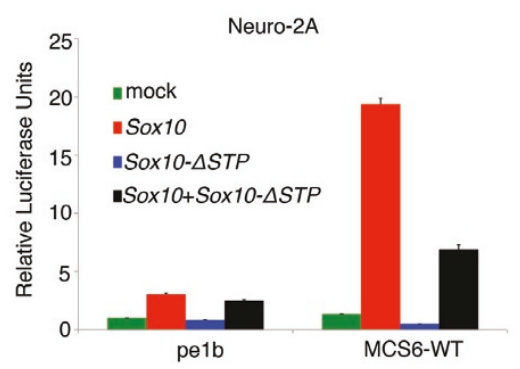

I

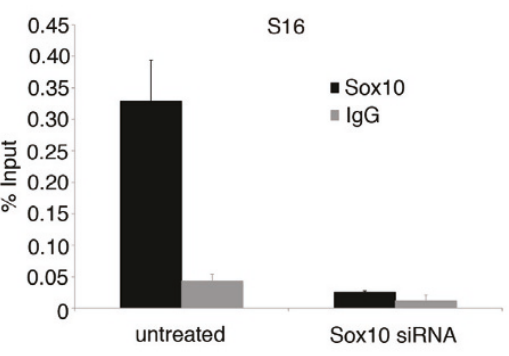

Figure 2 Sox 10 is necessary and sufficient for the activity of ERBB3_MCS6 in vitro. (A) Luciferase activities of wild-type ERBB3_MCS6 and TFBS mutations in ERBB3_MCS6 in melan-A cell line. The position of each TFBS in ERBB3_MCS6 is shown on top and the corresponding mutation in each TFBS is shown next to the luciferase value for that construct. ${ }^{*}$ indicates statistical significance. (B) Luciferase activity of WT ERBB3_MCS6 in melan-A cells in mock-transfected cells and in cells with transient Sox10 and Ap2 knockdown. (C) Western blot to confirm knockdown of Sox10 and Ap2 protein upon siRNA treatment. Tubulin antibody was used as a loading control. (D) Western blot showing Erbb3 protein levels in melan-a cells upon transient transfection with Sox10 and Ap2 siRNA or mock-transfected cells. Tubulin antibody was used as a loading control. (E) Luciferase activity of ERBB3_MCS6 upon knockdown of Sox10 in S16 cells using a dominant negative SOX10 mutant (E189X) under a CMV prmoter. (F) Luciferase assay of WT and SOXE-2m ERBB3_MCS6 in Neuro2A cells when transiently co-transfected with an empty expression vector (pcDNA.31) and Sox10 cDNA. Cell lysates were collected 24 hours post transfection. (G) Luciferase assay of WT ERBB3_MCS6 in Neuro2A cells when transiently co-transfected with equal amounts of WT and Sox10- $\triangle S T P$ CDNA either individually or in combination. Cell lysates were collected 24 hours post transfection. All luciferase values are normalized to renilla internal control and shown as fold-change compared to promoter only construct (pe1B) with standard deviation. (H) Real-time PCR of Erbb3 transcript levels upon expression of WT and Sox10- $\triangle S T P$ cDNA individually and in combination. Values are normalized to $18 \mathrm{~s}$ internal control and are shown as fold-change compared to promoter only construct (pcDNA3.1) with standard error. (I) Real-time PCR of ChIP against Sox10 in untreated S16 cells and in S16 cells treated with Sox10 morpholino. Black bar indicates enrichment upon Sox10 ChIP whereas grey bar indicates enrichment with non-specific lgG. Error bars indicate standard deviation of technical replicates in real-time PCR. 
together, these experiments demonstrate that Sox10 is necessary for the expression of $E r b b 3$ and for enhancer activity of ERBB3_MCS6, consistent with its predicted role in modulating Erbb3. Knockdown of Ap2 in melana cells also led to a significant reduction in ERBB3_MCS6 transcriptional activity (Figure 2B). Furthermore, $A p 2$ knockdown also reduced endogenous Erbb3 protein levels (Figure 2D), consistent with previous evidence for a role for $A P 2$ in regulating $E R B B 3$ $[35,36]$.

\section{Sox10 is sufficient for ERBB3_MCS6 enhancer activity and Erbb3 expression}

Given that Sox10 is necessary for ERBB3_MCS6 enhancer activity, we next tested whether Sox10 is also sufficient for ERBB3_MCS6 activity. We transiently cotransfected mouse neuroblastoma cells (Neuro2A) with the ERBB3_MCS6 luciferase vector and with either a construct expressing wild-type Sox10 cDNA under the control of a CMV promoter (pcDNA3.1) or with an empty expression vector. Neuro2A cells were selected due to low expression levels of Sox 10 and Erbb3 and because this cell line has been previously used to study the interaction between Sox10 and Erbb3 [10]. In the presence of Sox10, the transcriptional activity of ERBB3_MCS6 increased almost 25-fold, compared to cells co-transfected with the empty vector (Figure $2 \mathrm{~F}$ ). In the absence of Sox10, ERBB3_MCS6 does not display significant transcriptional activity in Neuro2A cells. Further, consistent with a role for Sox10 in directly regulating MCS6, mutation of the SOXE-2 site abrogates the increase in transcriptional activity. Similar results were also seen when human SOX10 cDNA was used in co-transfection experiments in NIH3T3 cells (Additional file 2, Figure S2). On the other hand, $A P 2$ was unable to significantly transactivate ERBB3_MCS6 in NIH3T3 cells (Additional file 2, Figure S2). Also, when added in combination with $S O X 10$, it was unable to increase transcriptional activity of ERBB3_MCS6 in a manner that is more than additive when compared to SOX10 and AP2 independent transactivation, further suggesting that $A P 2$ and SOX10 do not interact to regulate ERBB3_MCS6.

To further investigate the role of Sox10 in regulating ERBB3_MCS6, we next determined whether established disease causing mutations in Sox10 might in part mediate their regulation of Erbb3 via ERBB3_MCS6. To this end, we used a Sox10 mutant cDNA expression construct (c.928_929ins), hereby referred to as Sox10- $\triangle S T P$ to transactivate ERBB3_MCS6. This mutant was first discovered in the Dom mouse model of Hirschsrpung disease $[20,21]$ and has a single nucleotide (Guanine) insertion at nucleotide 929 of the coding sequence, leading to a translational frameshift. The resulting protein possesses an intact DNA binding domain but 99 novel nucleotides replace the putative activation domain. The resulting protein inhibits the activity of wild-type Sox 10 in a dominant negative fashion. Co-transfection of ERBB3_MCS6 with Sox10- $\triangle S T P$ does not result in ERBB3_MCS6 activity (Figure 2G). Additionally, $\triangle \mathrm{STP}$ also compromises the transactivation of ERBB3_MCS6 by wild-type Sox10 as seen in cells co-transfected with both the wild-type and the mutant forms of Sox10. This implies that ERBB3_MCS6 plays an important role in the regulation of Erbb3 by Sox 10 .

If ERBB3_MCS6 were indeed an important link in the regulation of Erbb3 expression by Sox10, we would expect the overexpression of wild-type Sox10, but not the Sox10- $S T T P$ mutant, to increase endogenous transcript levels of Erbb3. In fact, when we examined the levels of Erbb3 transcript in Neuro2A cells upon Sox10 overexpression, we found that it was seven-fold higher than Erbb3 transcript levels in the absence of Sox 10 (Figure 2H). Furthermore, Sox 10- $\triangle S T P$ was unable to increase Erbb3 transcript levels and it also impeded the ability of the wild-type protein to increase Erbb3 transcript levels. An increase in total Sox 10 transcript levels upon overexpression was verified via real-time PCR (Additional file 3, Figure S3). Therefore, this implies that Sox10 is sufficient for the transcriptional activation of Erbb3.

\section{Sox10 physically binds ERBB3_MCS6}

We next used electrophoretic mobility shift assays to determine if ERBB3_MCS6 physically binds protein in a manner dependent on the SOXE-2 motif. We generated 50 bp probes that span the SOXE-2 binding site and labeled the probes with biotin. We then incubated the probes with nuclear extract derived from melan-a cells and subjected the reactions to gel electrophoresis. As seen in Additional file 4, Figure S4, a factor in the melan-a nuclear extract bound the probe and retarded its migration in the gel, causing a shift. This binding was successfully competed away by using increasing amounts of unlabeled probe. To determine whether this binding was specific to the SOXE-2 binding site, we next mutated the SOXE-2 binding site and incubated unlabeled mutant probe with nuclear extract. As is seen in lane 5 of Additional file 4, Figure S4, the mutant cold probe was unable to compete away the binding, thus indicating that the gel shift seen is specific to binding at the SOXE-2 binding site. In order to assay whether Sox10 directly binds to ERBB3_MCS6, we used chromatin immunoprecipitation followed by quantitative PCR using an antibody directed against Sox10 in S16 cells. As shown in Figure 2I, the rat ortholog of ERBB3_MCS6 is enriched in Sox10 immunoprecipitated samples but not in IgG immunoprecipitated samples. This binding is specific to Sox10 as knocking down 
Sox10 protein levels in S16 via siRNAs caused a loss of enrichment of ERBB3_MCS6 upon Sox10 immunoprecipitated in these cells. Collectively, this implies that Sox10 directly binds ERBB3_MCS6.

\section{ERBB3_MCS6 directs reporter expression in a neural crest specific manner reminiscent of erbb3b expression in zebrafish}

In order to confirm that ERBB3_MCS6 is an enhancer of $E R B B 3$ in vivo, we generated stable transgenic zebrafish lines of ERBB3_MCS6 driving an eGFP reporter using a Tol2 based zebrafish transgenesis assay [39]. Briefly, we cloned ERBB3_MCS6 upstream of a $c f o s$ minimal promoter driving enhanced green fluorescent protein (eGFP). We then injected this sequence into 1-2 cell zebrafish embryos, screened G0 embryos for vector integration, and evaluated their offspring for germ line reporter expression. Reporter expression was assayed in four independent transgenic lines and compared to previously published patterns of endogenous Erbb3/erbb3b expression [10-12,14]. Consistent with its strong in vitro regulatory potential, ERBB3_MCS6 drove reporter expression in NC populations as early as 24 hours post fertilization (hpf) in transgenic zebrafish in a manner reminiscent of erbb3b expression (Figure 3A, B). Expression was noted in cranial neural crest, migratory and pre-migratory crest, all regions where $E r b b 3 / e r b b 3 b$ is known to be expressed (Figure 3A, B) [11]. At $48 \mathrm{hpf}$, expression was noted in the dorsal root ganglia, posterior lateral line ganglia and in Schwann cell precursors, consistent with known Erbb3/erbb3b expression (Figure 3C) $[12,13]$. Finally, at $72 \mathrm{hpf}$ expression was noted in cells that are consistent in shape and position with mature oligodendrocytes (Figure 3D) [40]. Therefore, ERBB3_MCS6 drives reporter expression in vivo in neural crest cells and its derivative tissues in a manner consistent with erbb3b expression. Two other sequences, ERBB3_MCS1 and MCS4 also drove consistent expression in multiple founders in $\mathrm{NC}$-derived populations consistent with Erbb3/erbb3b expression (Additional file 5, Figure S5). Although ERBB3_MCS1 did not show significant enhancer activity in vitro, or H3K4me1 marks, it demonstrated expression in vivo in a manner consistent with Erbb3 expression. This sequence directed reporter expression starting at $48 \mathrm{hpf}$ in the mesencephalon, olfactory bulbs, cranial ganglia and pharyngeal arches, all regions of demonstrated Erbb3 expression [14,41]. ERBB3_MCS1 also directed expression in the midbrain-hindbrain boundary and in the hindbrain, regions where Erbb3 function has been shown to be essential [9] (Additional file 5, Figure S5A, B, C). Furthermore, this element directed expression in cells lining the anterior lateral line neurons and the epiphysis (data not shown). Consistent with its strong in vitro regulatory potential and H3K4me1 marks, ERBB3_MCS4 directed reporter expression in vivo. ERBB3_MCS4 drove eGFP expression in vivo, beginning at $24 \mathrm{hpf}$ in the mesencephalon, the hindbrain and the pharyngeal arches. It also directed expression in the myotome consistent with endogenous Erbb3 expression $[10,11]$ (Additional file 5, Figure S5D, E, F). This suggests that ERBB3_MCS1, MCS3 and MCS6 are all NCdirected enhancers of ERBB3.

\section{SOXE-2 motif is necessary for the activity of ERBB3_MCS6 in vivo in zebrafish}

To determine the contribution of the SOXE-2 motif to the proper expression of ERBB3_MCS6 in vivo, we generated stable transgenic zebrafish lines with ERBB3_MCS6 harbouring a mutation in the SOXE-2 motif. We identified three independent stable transgenic zebrafish lines with the SOXE-2m ERBB3_MCS6 construct directing eGFP expression. eGFP expression was inconsistent among these lines. This may either be a direct result of position effect based on the position of integration of the vector, or mutation of the SOXE-2 motif may make the sequence more amenable to position effect due to loss of proper regulatory control. In one of the three lines, eGFP expression was not seen at $24 \mathrm{hpf}$ whereas in the remaining two lines eGFP expression was qualitatively similar to expression directed by the WT ERBB3_MCS6 construct at 24 hpf (Figure 3E, F). Additionally, mutation of this site may yield quantitative differences in eGFP expression between the WT and mutant constructs. Technical reasons such as variation in transgene copy number and position effect preclude the use of this assay to evaluate such differences. Therefore, we cannot exclude the possibility that additional elements within ERBB3_MCS6 may be sufficient for its expression in NC in the early embryo. However, at $48 \mathrm{hpf}$ and $72 \mathrm{hpf}$, expression driven by the mutant construct was qualitatively different across all founders as compared to expression directed by the WT construct. Expression was lost from dorsal root ganglia cells and Schwann cells in 48 hpf SOXE-2m ERBB3_MCS6 transgenic embryos. Expression domains were gained within these mutants in the notochord and cells of the ventral spinal cord (Figure $3 G$ and data not shown). Furthermore, at $72 \mathrm{hpf}$ expression was not seen in oligodendroglial cells in SOXE-2m ERBB3_MCS6 transgenic embryos (Figure $3 \mathrm{H}$ ). However, at both $48 \mathrm{hpf}$ and 72 hpf, expression in the ectomesenchymal derivatives of the NC such as in the pharyngeal arches was maintained in the mutant transgenic fish (data not shown). This is in agreement with a known role for Sox10 in non-ectomesenchymal but not in ectomesenchymal NC populations in the zebrafish [42]. This suggests that additional elements within ERBB3_MCS6 are sufficient for the 


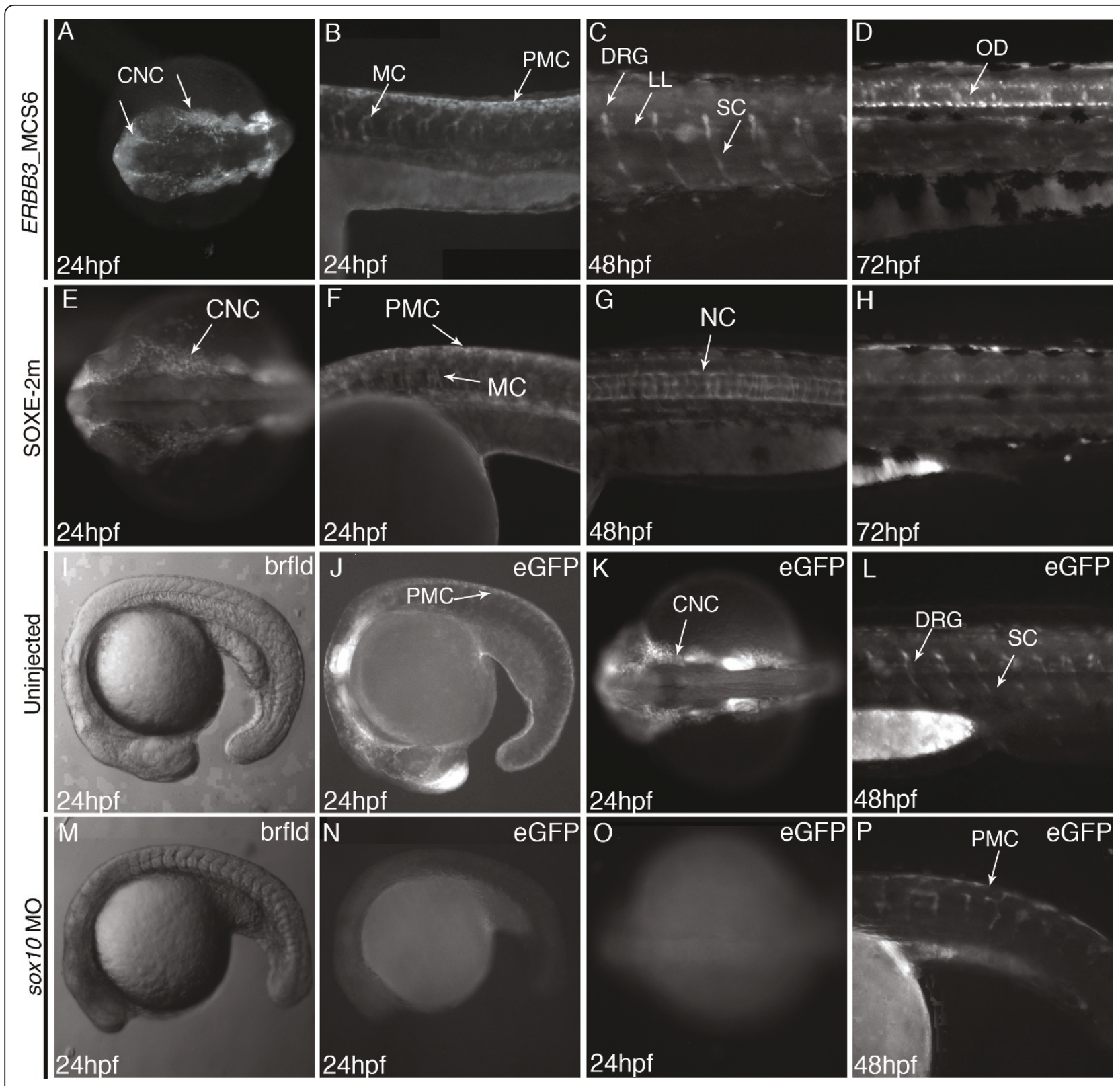

Figure 3 ERBB3_MCS6 drives reporter expression in vivo in a pattern similar to erbb3b and under the control of sox10. (A-D) Expression pattern of ERBB3_MCS driving eGFP in G1 transgenic 24-72hpf zebrafish embryos. Arrows indicate tissues where expression was noted in multiple founders. (E-H) eGFP expression pattern seen in multiple founders of SOXE-2m ERBB3_MCS6 transgenic fish. Although expression is similar to WT ERBB3_MCS6 at 24hpf, expression differs at later stages of development. (I-P) Results of morpholino knockdown of sox10 in ERBB3_MCS6 transgenic embryos. (I-L) Normal expression of ERBB3_MCS6 in uninjected transgenic embryos at 24-48hpf. (M-P) Loss of reporter expression in embryos injected with a Sox10 morpholino at 24hpf and appearance of disorganized GFP expressing NC cells at 48hpf. Abbreviations: cranial neural crest (CNC), migratory crest (MC), pre-migratory crest (PMC), dorsal root ganglia (DRG), posterior lateral line (PLL), Schwann cells (SC), oligodendrocytes (OD) and notochord (NC).

expression of this sequence in ectomesenchymal derivatives of the NC. Therefore, the SOXE-2 motif is important for the proper expression of ERBB3_MCS6 in nonectomesenchymal neural crest derivatives such as dorsal root ganglia and Schwann cells, but not in premigratory and migratory crest cells or ectomesenchymal NC derivatives. However, based on the diminution and not complete abrogation of ERBB3_MCS6 activity in vitro, we expect that mutation of this site may produce quantitative effects on the expression of ERBB3_MCS6, which cannot be assayed by zebrafish transgenesis. Therefore, to address the role of Sox10 in regulation 
ERBB3_MCS6 in vivo, we used morpholinos analysis in zebrafish.

\section{sox10 is necessary for the activity of ERBB3_MCS6 in vivo in zebrafish}

In order to determine whether Sox10 is required for the enhancer activity of ERBB3_MCS6 in vivo similar to its requirement in vitro, we used morpholinos against sox10 in the stable transgenic ERBB3_MCS6 zebrafish to assay the effect of sox10 depletion on ERBB3_MCS6 activity. Using a previously published translation-blocking morpholino against the zebrafish sox 10 gene product, we knocked down levels of sox10 in the ERBB3_MCS6 transgenic zebrafish [43]. Depletion of sox10 in zebrafish produces a phenocopy of the colourless $\left(\operatorname{sox} 10^{-1-}\right)$ zebrafish mutant phenotype, which has been studied in detail [42]. Although NC cells form upon sox10 depletion, they accumulate in the premigratory position and fail to migrate and differentiate appropriately into their subsequent lineages $[42,43]$. Thus, if sox10 were necessary for ERBB3_MCS6 expression, we would expect to see a loss of ERBB3_MCS6 driven reporter expression in premigratory $\mathrm{NC}$ as well as a subsequent loss of expression in NC derived cell types such as Schwann cells and DRG due to the loss of these populations. Consistent with our expectation, upon knocking down sox10, we noted a complete loss of ERBB3_MCS6 activity in the developing $\mathrm{NC}$ in one-day old embryos (Figure 3N,O). This is in sharp contrast to the ERBB3_MCS6 driven reporter expression seen in the premigratory and migratory $\mathrm{NC}$ of uninjected embryos (Figure 3J,K). Since the sox10 morpholino produces embryos with a range of phenotypes, in embryos that show a weaker phenotype, we noticed a reduced number of eGFP expressing premigratory NC cells and highly reduced numbers of migrating crest at $24 \mathrm{hpf}$, consistent with a role for Sox10 in regulation ERBB3_MCS6 (Additional file 6, Figure S6C, D). eGFP expression in the cranial NC was affected to a lesser degree in these embryos (Additional file 6, Figure S6C). However, eGFP expression in $24 \mathrm{hpf}$ embryos was noted in only 6/130 injected embryos (4.6\%), which is in sharp contrast to the $27 / 60$ uninjected $(45 \%)$ embryos that showed eGFP expression at $24 \mathrm{hpf}$. By $48 \mathrm{hpf}$, ERBB3_MCS6 driven eGFP expression was noted in morpholino injected embryos in cells that are consistent in position with premigratory NC (Figure 3P). Expression was also noted in some migratory $\mathrm{NC}$, however, these cells are highly disorganized as compared to the eGFP expressing cells seen in uninjected embryos consistent with migratory crest previously described for this model (Figure 3L, P) [42,43]. eGFP expression persists in these non-migrating $\mathrm{NC}$ cells upto $96 \mathrm{hpf}$ (data not shown) but is not seen in NC derived cells. Therefore, this suggests that sox10 is necessary for the timely and proper expression of ERBB3_MCS6 in neural crest cells and its derivative lineages in vivo in zebrafish.

\section{Discussion}

Although ERBB3 is an important gene in the development and differentiation of a range of NC-derived populations, not much is known about the sequences and factors modulating its transcriptional output. Sox10, however, has been shown to influence Erbb3 levels [10], although, whether this regulation is direct or indirect was unknown. In this study, we identify novel regulatory enhancers of $E R B B 3$ that direct expression in NC derived cell lines and exhibit epigenetic marks consistent with enhancer activity. One of these enhancers, ERBB3_MCS6 directs the strongest reporter expression in vitro and a broad overlap with endogenous erbb3b expression in vivo in zebrafish. We demonstrate that ERBB3_MCS6 is dependent upon and is responsive to Sox10 both in vitro and in vivo. Furthermore, we also demonstrate that Sox10 is both necessary and sufficient in vitro for endogenous Erbb3 expression. Finally, we demonstrate that Sox10 physically binds to ERBB3_MCS6, suggesting that SOX10 mediates its effect on $E R B B 3$, at least in part, directly through ERBB3_MCS6.

Using a combination of sequence constraint, in vitro luciferase and chromatin immunoprecipitation assays, and in vivo zebrafish transgenesis, we have identified three functional enhancers of ERBB3-ERBB3_MCS1, MCS4 and MCS6. Although ERBB3_MCS1 did not significantly drive luciferase activity in vitro or exhibit enrichment of H3K4me1 marks, it drove reporter expression in transgenic zebrafish embryos in a manner consistent with endogenous erbb3b expression. Furthermore, although elements ERBB3_MCS2, MCS3 and MCS7 showed increased luciferase activity in vitro, they did not exhibit NC-directed enhancer activity in vivo. This suggests that although in vitro assays are useful in assaying the regulatory potential of enhancer sequences, they represent a single stage and cell type in development as compared to the full spectrum of NC derivatives that can be assayed in vivo and can potentially overlook regulatory sequences, which may be active at different stages or cell types or may attribute activity to sequences that are not biologically functional. Similarly, comparison of H3K4me1 marks and enhancer activity in the elements tested demonstrates that H3K4me1 is not sufficient to distinguish between active and inactive enhancers. ERBB3_MCS5, MCS7 and MCS8 showed very high levels of H3K4me1, but did not direct transcriptional activity in vitro or in vivo, suggesting that additional epigenetic or transcriptional marks may be necessary to distinguish them from active enhancers. 
These data are consistent with recent reports that suggest that additional epigenetic marks such as H3K27ac may be necessary to distinguish between H3Kem1 marked active and poised enhancers [28,29].

The intronic enhancer ERBB3_MCS6 demonstrated high transcriptional activity in vitro and also exhibited H3K4me1 marks. Furthermore, it directed expression almost exclusively, and broadly, in developing NC populations. The role of AP2 in regulating ERBB3_MCS6 remains unclear. Mutation of the AP2 binding site does not significantly reduce transcriptional activity of $E R B B 3$ MCS6, suggesting that it is not required for the activity of ERBB3_MCS6. Furthermore, ectopic expression of $A P 2$ does not increase transcriptional activity of ERBB3_MCS6 (Additional file 2, Figure S2). However, knockdown of $A p 2$ caused a significant decrease in transcriptional activity of ERBB3_MCS6 (Figure 2B). Therefore, although $A P 2$ may be necessary for the activity of $E R B B 3$ 3 MCS6, it is not sufficient for transcriptional activity of ERBB3_MCS6, suggesting that it may act in concert with other transcription factors to mediate its effect on ERBB3_MCS6. Based on the results of our assays, it does not seem that AP2 interacts with SOX10 to regulate ERBB3_MCS6, although we cannot exclude the possibility that the contribution of $A P 2$ to the regulation of ERBB3_MCS6 may be rate limited by and secondary to regulation by $S O X 10$. Therefore, further examination will be required to determine how $A P 2$ may regulate ERBB3_MCS6 and what, if any, co-factors may mediate this regulation. Since $A P 2$ is known to regulate $E R B B 3$, it is not surprising that we see a decrease in Erbb3 protein levels upon $A p 2$ knockdown in melan-a cells $[35,36]$, however whether this decrease is mediated in part via ERBB3_MCS6 will require further investigation. Similarly, it will also be interesting to determine what, if any, other TFs may be involved in regulating $E R B B 3$ via ERBB3_MCS6 by expanding the search for functionally important sequence motifs therein.

However, by focusing our investigation on the role of SOX10 in regulating ERBB3_MCS6, we show that it is directly responsive to $S O X 10$. Although there were two potential Sox10 binding sites in ERBB3_MCS6, only one of them, SOXE-2 was required in our in vitro assays. This site is perfectly conserved across mammals and mutation of the site causes a loss of Sox 10 responsiveness of ERBB3_MCS6. Furthermore, mutation of the SOXE-2 site affects the expression of ERBB3_MCS6 in non-ectomesenchymal $\mathrm{NC}$ derivatives in zebrafish, but not in ectomesenchymal derivatives of $\mathrm{NC}$ or in early premigratory and migratory NC. Although quantitative differences in expression of ERBB3_MCS6 due to the SOXE-2 mutation cannot be ruled out as they are below the threshold of detection by the zebrafish transgenesis assay, this data suggests that other motifs within ERBB3_MCS6 may be sufficient for its expression in early NC and ectomesenchymal derivatives. However, using additional in vitro and in vivo assays, we established an important role for Sox10 in regulating ERBB3_MCS6 via the SOXE-2 motif. Knockdown of Sox10 abrogates ERBB3_MCS6 activity, whereas ectopic expression of Sox10 induces ERBB3_MCS6 response, demonstrating that Sox10 is both necessary and sufficient for ERBB3_MCS6 transcriptional activity. Similarly, we also show that Sox10 is both necessary and sufficient for Erbb3 expression. This is consistent with previous data suggesting that Erbb3 is under the control of Sox 10 [10]. Using gel-shift and chromatin immunoprecipitation assays, we also demonstrate direct binding of Sox10 to ERBB3_MCS6, most likely mediated through the SOXE2 site. Finally, we use morpholino analysis in ERBB3_MCS6 transgenic zebrafish to demonstrate a requirement for Sox10 in the proper expression of this enhancer. Therefore, our data suggest that $E R B B 3 \_$MCS6 may play a critical role in mediating the regulation of ERBB3 by SOX10.

\section{Conclusions}

In conclusion, we have identified three transcriptional enhancers at the ERBB3 locus, whose expression domains overlap NC-derived populations. We show that one such enhancer (ERBB3_MCS6) directs reporter expression broadly in NC cells and, like $E R B B 3$, is directly responsive to Sox10, implying that Sox10 may act through this enhancer to regulate ERBB3 transcription in the NC.

\section{Methods}

Fish husbandry and transgene and morpholino injections

Zebrafish were bred and maintained as previously described $[44,45]$. Microinjections were carried out as previously described [39]. Briefly, eGFP expression vectors were injected into $1-2$ cell stage embryos $(n \geq 300)$. Reporter expression was assayed between $24 \mathrm{hpf}-5 \mathrm{dpf}$ and embryos with consistent GFP expression were selected and raised to adulthood and founders were identified. For morhpholino experiments, previously published sox10 morpholino was ordered from Gene Tools (Philomath, OR) [43]. 6.6 ng of the morpholino was injected into each embryo by microinjection. Embryos were analyzed and imaged using a Carl Zeiss Lumar V12 Stereo microscope with AxioVision version 4.8 software. Transgenic lines for ERBB3_MCS1, ERBB3_MCS4 and ERBB3_MCS3 are listed at the Zebrafish International Resource Center (ZIRC) (http://zebrafish.org) under allele designations JH112, JH113 and JH114 respectively. 


\section{Identification of conserved non-coding sequences and transcription factor binding sites}

Conserved sequences were identified at the human $E R B B 3$ locus and upstream and downstream intergenic regions (chr12:54,724,274-54,784,370) using the PhastCons custom track on the UCSC Genome Browser (http://genome.ucsc.edu) on genome build hg18. We used the 17-way MutiZ alignment to identify the most conserved sequences within the introns of the gene and within intergenic regions surrounding the gene. The coordinates of the MCSs and the primers used to amplify them are shown in Additional file 7, Table S1.

Fasta format DNA sequence of ERBB3_MCS6 from the genome browser was used to query MatInspector [46], MATCH 1.0 and Jaspar databases for identifiable TFBS using default settings.

\section{Vector construction and mutagenesis}

Expression vectors were constructed using Gateway Technology (Invitrogen, Carlsbad, CA). The desired genomic regions were amplified by with attB-flanked primers and recombined into the pDONR221 vector. Successful recombination was confirmed by sequencing. Next, entry clones were recombined into the destination vectors pLGF-E1b for luciferase assays and pT2cfosGW for zebrafish injections $[25,47]$.

ERBB3_MCS6 was mutated using the QuikChange II XL Site-directed mutagenesis kit (Stratagene, La Jolla, CA). Mutagenesis primers were designed to change the potential transcription factor binding sites (TFBS) to Hpa1 restriction sites using the QuikChange Primer Design tool. Primers are included in Additional file 8, Table S2.

Sox10-pcDNA3.1 and Sox10- $\triangle S T P$-pcDNA3.1 were cloned using Sox10-pCMV and Sox10- $S T P-$ pCMV as templates. Coding sequence for human SOX10 and AP2 for transactivation experiments was amplified by PCR from I.M.A.G.E clone MGC-3510 and MGC-22117 respectively and cloned into pcDNA3.1 (Invitrogen, Carlsbad, CA) using In-Fusion PCR Advantage Cloning Kit (Clontech, Mountain View, CA). The PCR primers used were designed using In-Fusion Primer Design tool and are shown in Additional file 9, Table S3. Successful cloning was verified by sequencing.

The E189X Sox10 cDNA cloned into a pCMV promoter was a kind gift from Ken Inoue, Jim Lupski and Michael Wegner [23,38].

\section{Cell culture and transfection}

Immortalized melanocytes (melan-a) and immortalized Schwann cells were maintained as described $[48,49]$. NIH 3 T3 cells were grown in 10\% FCS in DMEM under standard conditions. Neuro2A cells were grown in $10 \%$ FCS in MEM under standard conditions.

\section{Luciferase assay}

melan-a cells were plated in 24-well plates 24 hours prior to transfection at a density of $4 \times 10^{\wedge} 4$ cells/well. $400 \mathrm{ng}$ of the luciferase reporter plasmids were cotransfected with $8 \mathrm{ng}$ of CMV-RL renilla expression vector (Promega, Madison, WI). 48 hours after transfection, cell lysate was collected and assayed using the DualLuciferase Reporter Assay System (Promega, Madison, WI). For siRNA knockdown, 200 ng of luciferase reporter vectors were cotransfected with 5 pmol of each siRNA pool to a total of $10 \mathrm{pmol} /$ well of a 24-well plate. Where appropriate, scrambled siRNA was added to maintain a final concentration of $10 \mathrm{pmol} /$ well of siRNA.

Neuro2A cells were plated in 24-well plates 24 hours prior to transfection at a density of $5 \times 10^{\wedge} 4$ cells/well. $400 \mathrm{ng}$ of luciferase reporter plasmids were cotransfected with $200 \mathrm{ng}$ of Sox 10-pcDNA3.1 and $\triangle S T P$ Sox10-pcDNA3.1. Where appropriate, empty pcDNA3.1 vector was added to maintain a final concentration of $800 \mathrm{ng} /$ well of DNA. $8 \mathrm{ng}$ of CMV-RL were added to each well. Cell lysate was collected 24 hours after transfection and assayed as mentioned above. Luciferase assays were carried out using a Tecan GENiosPro machine. All assays were performed in triplicate and repeated in at least two independent experiments.

For luciferase assays in S16 cells, $1 \times 10^{\wedge} 4$ cells were plated in 96-well plates 24 hours prior to transfection with luciferase vectors. Each transfection reaction included $200 \mathrm{ng}$ of experimental and control luciferase expression constructs and $2 \mathrm{ng}$ of a renilla expression construct to control for transfection efficiency and cell viability. Cells lysates were collected 48 hours after transfection and luciferase assays were carried out with the Dual-Luciferase Assay System (Promega, Madison, WI) and analyzed on a Glomax Multi-Detection System (Promega, Madison, WI).

\section{siRNA knockdown}

ON-TARGETplus SMARTpool siRNA was ordered against mouse Sox 10 and Tfap $2 a$ from Dharmacon (Lafayette, CO). Knockdown was achieved by using 5 pmol of each siRNA in a 24-well transfection format.

\section{Western blot}

Cells were trypsinized and washed twice in 1XPBS and resuspended in $2 \mathrm{X}$ Incomplete Lamelli buffer and passed through a QiaShredder (Invitrogen, Carlsbad, CA) to obtain whole cell lysate. Protein was quantified and run on a $10 \%$ Mini Protean TGX gel (Biorad, Hercules, CA), transferred onto a nitrocellulose membrane and blocked overnight in 5\% non-fat dry milk block. Sox10 antibody was used at a dilution of $1.5 \mathrm{ng} / \mathrm{ul}$ (MAB2864, R\&D Biosystems, Minneapolis, MN), AP2 antibody was used at 
1:500 (ab52222, Abcam, Cambrigde, MA), ErbB3 antibody was used at 1:500 (sc285, Santa Cruz Biotechnology, Santa Cruz, CA) and anti-tubulin at 1:3000 (CP06, Calbiochem, San Diego, CA). HRP-conjugated secondary was used and antibody binding was visualized using SuperSignal West Dura Extended Duration Substrate (34076, Thermo Fischer, Rockford, IL). Membranes were stripped using Restore PLUS Western Blot Stripping Buffer (46430, Thermo Fischer, Rockford, IL

\section{RNA extraction, CDNA synthesis and Real-time PCR}

Neuro2A cells were plated in 6-well dish at a density of $2.5 \times 10^{\wedge} 5$ cells/well using Lipofectamine 2000 (Invitrogen, Carlsbad, CA). A total of 2 ng of Sox10-pcDNA3.1 and $\triangle S T P$-Sox 10-pcDNA3.1 were transfected into the cells and RNA was collected 24 hours later using the RNeasy Mini Kit (Qiagen, Valencia, CA) using manufacturers instructions. Subsequently, cDNA was synthesized using the SuperScriptIII First Strand Synthesis System for RT-PCR (Invitrogen, Carlsbad, CA). Real-time PCR was then performed in triplicate using the Universal Gene Expression Master Mix (ABI, Carlsbad, CA) and the PrimeTime qPCR Assay designed against mouse Sox10 and ErbB3. Real-time PCR and analysis were performed on the Opticon2 (Biorad, Hercules, CA).

\section{Electrophoretic mobility shift assay}

Probes spanning the SOXE-2 binding site were designed (Additional file 10, Table S4) and labeled with the Biotin 3' End DNA Labeling Kit (Pierce, Rockford, IL) according to manufacturers instructions. Nuclear extract was made from melan-a cells using the NE-PER Nuclear and Cytoplasmic Extraction Reagents (Pierce, Rockford, IL). EMSAs were performed using the LightShift Chemiluminescent EMSA Kit (Pierce, Rockford, IL). Briefly, 12.5 fmol of labeled probe was incubated with nuclear extract in the presence of binding buffer and $1 \mathrm{ug}$ of poly (dI.dC) in a $20 \mathrm{ul}$ reaction for $10 \mathrm{~min}$ at room temperature. For competition assays, 500 and 1000 molar fold excess of unlabeled probe was added. Products were run on precast polyacrylamide gels $(4-20 \%$ or 7.5\%) (Biorad, Hercules, CA) and signal was developed using the LightShift Chemiluminescent kit.

\section{Chromatin Immunoprecipitation (ChIP) and Real-time PCR}

ChIP was performed in melan-a cells as previously described [50] with some changes. Each ChIP experiment was performed with $\sim 1 \times 10^{8}$ cells. Alternative lysis buffers to those in the referenced protocol were used, as follows: Lysis buffer 1 (5 mM PIPES, $85 \mathrm{mM}$ $\mathrm{KCl}, 0.5 \% \mathrm{NP}-40$, and $1 \times$ Roche Complete, EDTA-free protease inhibitor), lysis buffer $2(50 \mathrm{mM}$ Tris- $\mathrm{HCl}, 10$ mM EDTA, 1\% SDS, and $1 \times$ Roche Complete, EDTAfree protease inhibitor), and lysis buffer 3 (16.7 mM
Tris- $\mathrm{HCl}, 1.2 \mathrm{mM}$ EDTA, $167 \mathrm{mM} \mathrm{NaCl}, 0.01 \%$ SDS, $1.1 \%$ Triton X-100, and $1 \times$ Roche Complete, EDTAfree protease inhibitor). Sonication was performed with a Bioruptor (Diagenode, Denville, NJ) with the following settings: high output; 30 second disruption; 30 second cooling; total sonication time of 35 min with addition of fresh ice and cold water to water bath every 10 minutes. 2 ug of antibody specific to H3K4me1 (ab8895, Abcam Cambridge, MA) and non-specific IgG (ab46540, Abcam Cambridge, MA) was used for immunoprecipitation. IP wash conditions were also adjusted from the above referenced protocol, as follows: Each IP was washed twice with low salt wash buffer $(0.1 \%$ SDS, $1 \%$ Triton X100, 2 mM EDTA, $20 \mathrm{mM}$ Tris- $\mathrm{HCl}, 150 \mathrm{mM} \mathrm{NaCl}$ ), twice with high salt wash buffer $(0.1 \%$ SDS, $1 \%$ Triton $\mathrm{X}-100,2 \mathrm{mM}$ EDTA, $20 \mathrm{mM}$ Tris- $\mathrm{HCl}, 500 \mathrm{mM} \mathrm{NaCl}$ ), twice with cold $\mathrm{LiCl}$ wash buffer $(0.25 \mathrm{M} \mathrm{LiCl}, 1 \% \mathrm{IGE}$ PAL CA630, 1\% deoxycholic acid (sodium salt), $1 \mathrm{mM}$ EDTA, $10 \mathrm{mM}$ Tris- $\mathrm{HCl}$ ), and rinsed once with PBS, $\mathrm{pH}$ 7.4. Immunoprecipitated DNA was analyzed by realtime PCR using SYBR Green (ABI, Carlsbad, CA) and primers shown in Additional file 11, Table S4 and performed and analyzed on an Opticon2 (Biorad, Hercules, CA) using the \% Input method. Primer sequences for PCR are given in supplementary table 5 .

ChIP in S16 cells was performed as previously described [51]. The antibodies used were anti-Sox10 (sc17342X, Santa Cruz Biotechnology, Santa Cruz, CA) and control anti-goat IgG (sc-2808, Santa Cruz Biotechnology, Santa Cruz, CA). ChIP was analyzed in duplicate by quantitative PCR and analyzed by the \% Input method. Primers used for PCR are shown in Additional file 11, Table S4.

\section{Additional material}

Additional file 1: Figure S1 - Identification of putative neural crest
transcription factor binding sites in ERBB3_MCS6. Sequence of
intronic neural crest enhancer ERBB3_MCS6 showing the location of
putative transcription factor binding sites (TFBS) identified using multiple
TFBS search programs. SOXE-2 adheres to the SOXE binding consensus
sequence.
Additional file 2: Figure S2 - Transcriptional transactivation of
ERBB3_MCS6 by SOX10 and AP2. Luciferase assay of wild type
ERBB3_MCS6 in NIH3T3 cells when transiently co-transfected with equal
amounts of an empty expression vector (pCDNA.31), SOX10 CDNA or AP2
CDNA. Cell lysates were collected 24 hours post transfection. All values
are normalized to a renilla internal control and shown as fold-change
compared to the promoter only construct (pe1B) with standard
deviation.
Additional file 3: Figure S3 - Increase in Sox10 transcript levels
upon Sox10 overexpression. Real-time PCR results showing an increase
in WT and mutant Sox10 CDNA upon transient transfection of WT and
Sox10- $\triangle S T P$ CDNA in Neuro2A cells. Values are normalized to an 185
internal control and shown as a fold-change compared to the promoter
only construct (pCDNA3.1) with standard error.
Additional file 4: Figure S4 - A nuclear protein within melan-a cells
binds SOXE2. EMSA demonstrating binding of ERBB3_MCS6 to a protein


in melan-a nuclei at the SOXE-2 site. Nuclear extract binds free probe (Lane 1) and shifts it upwards (Lane 2). Addition of 500X (Lane 3) and 1000X (Lane 4) molar excess of unlabeled probes competes shift. Addition of cold unlabeled probe with a mutation in the SOXE-2 binding site does not compete away the shift (Lane 5).

Additional file 5: Figure S5 - ERBB3 MCS1 and ERBB3 MCS4 drive reporter expression in vivo in a pattern similar to erbb3b. (A-J) Expression pattern of the indicated MCS driving eGFP in G1 transgenic 24-72hpf zebrafish embryos. Arrows indicate tissues where expression was noted in multiple founders. Abbreviations: mesencephalon (M), hindbrain (HB), olfactory bulb (OB), pharyngeal arches (PA), cranial ganglia, posterior lateral line ganglia (PLLg).

Additional file 6: Figure S6- Range of eGFP phenotypes in ERBB3_MCS6 transgenic fish upon sox10 morpholino injection. (A-B) eGFP expression driven by ERBB3_MCS6 in uninjected fish at 24hpf. Expression is noted in cranial neural crest (CNC), premigratory NC (PMC) and migratory crest (MC) (C-D) Fewer eGFP positive CNC (C) and PMC cells seen in sox10 morpholino injected transgenic embryos, and significantly reduced numbers of MC (D).

Additional file 7: Table S1-Coordinates of the ERBB3_MCS8 elements (human genome build hg18) and primers used for PCR amplification of each element.

Additional file 8: Table S2- Primers used for site-directed mutagenesis of ERBB3_MCS6

Additional file 9: Table S3- Primers used for cloning WT and mutant sox10 CDNA, AP2 and SOX10 CDNA.

Additional file 10: Table S4- Probes used for EMSA assay

Additional file 11: Table S5- Primers used for QPCR analysis of ChIP assay.

\section{Acknowledgements}

The authors would like to thank the Avramopoulos lab for their kind gift of Neuro2A cells and Seneca Bessling for her help with zebrafish husbandry. We would also like to thank Zachary Stine for critical scientific discussion and reading of the manuscript and also Grzegorz Burzynski for critical reading of the manuscript. Funding for this work comes from NIH/NIGMS and NIH/NINDS grants to ASM (GM071648 and NS062972), a NIGMS grant to SJ (GM056988), a NIH grant to JS (HD41590), a NIH/NINDS grant to AA (NS073748) and intramural NIH grants to WJP.

\section{Author details}

${ }^{1}$ McKusick-Nathans Institute of Genetic Medicine, The Johns Hopkins University School of Medicine, Baltimore, Maryland 21205, USA. ${ }^{2}$ Genetic Disease Research Branch, National Human Genome Research Institute, National Institutes of Health, Bethesda, Maryland 20892, USA. ${ }^{3}$ Department of Genetics, Washington University School of Medicine, St. Louis, Missouri 63110, USA. ${ }^{4}$ Department of Human Genetics, University of Michigan Medical School, Ann Arbor, Michigan 48109, USA. ${ }^{5}$ Department of Neurology, University of Michigan Medical School, Ann Arbor, Michigan 48109, USA. ${ }^{6}$ Program in Cellular and Molecular Biology; University of Wisconsin-Madison, Madison, Wisconsin 53705, USA. 'Department of Comparative Biosciences, University of Wisconsin-Madison, Madison, Wisconsin 53705, USA. ${ }^{8}$ Department of Molecular and Comparative Pathobiology, The Johns Hopkins University School of Medicine, Baltimore, Maryland 21205, USA.

\section{Authors' contributions}

MKP, SKL, WJP and ASM conceived of the study. MKP designed and performed the experiments, analyzed all data, and wrote the manuscript. XR and DG performed the ChIP experiment, JC participated in the luciferase assays, SKL contributed to the study design and data analysis, identified the MCS elements and cloned them into pe1B and PXIG vectors, ARM, KC and SJ participated in the creation and analysis of zebrafish transgenics, EAJ and JS did the chromatin immunoprecipitation assays in S16 cells, $\mathrm{CJH}$ and AA did the luciferase assays in S16 cells, WJP participated in the design and analysis of the study, ASM participated in the design and analysis of the study and helped write the manuscript. All authors read and approved the final manuscript.

\section{Competing interests}

The authors declare that they have no competing interests.

Received: 25 May 2011 Accepted: 14 June 2011 Published: 14 June 2011

\section{References}

1. Basch ML, Garcia-Castro MI, Bronner-Fraser M: Molecular mechanisms of neural crest induction. Birth Defects Res C Embryo Today 2004, 72(2):109-123.

2. Hou L, Pavan WJ: Transcriptional and signaling regulation in neural crest stem cell-derived melanocyte development: do all roads lead to Mitf? Cell Res 2008, 18(12):1163-1176.

3. McCallion AS, Chakravarti A: EDNRB/EDN3 and Hirschsprung disease type II. Pigment Cell Res 2001, 14(3):161-169.

4. Britsch S: The neuregulin-l/ErbB signaling system in development and disease. Adv Anat Embryol Cell Biol 2007, 190:1-65.

5. Carraway KL, Sliwkowski MX, Akita R, Platko JV, Guy PM, Nuijens A Diamonti AJ, Vandlen RL, Cantley LC, Cerione RA: The erbB3 gene product is a receptor for heregulin. J Biol Chem 1994, 269(19):14303-14306.

6. Guy PM, Platko JV, Cantley LC, Cerione RA, Carraway KL: Insect cellexpressed p180erbB3 possesses an impaired tyrosine kinase activity. Proc Natl Acad Sci USA 1994, 91(17):8132-8136

7. Carraway KL, Soltoff SP, Diamonti AJ, Cantley LC: Heregulin stimulates mitogenesis and phosphatidylinositol 3-kinase in mouse fibroblasts transfected with erbB2/neu and erbB3. J Biol Chem 1995, 270(13):7111-7116.

8. Wallasch C, Weiss FU, Niederfellner G, Jallal B, Issing W, Ullrich A: Heregulindependent regulation of HER2/neu oncogenic signaling by heterodimerization with HER3. EMBO J 1995, 14(17):4267-4275.

9. Erickson SL, O'Shea KS, Ghaboosi N, Loverro L, Frantz G, Bauer M, Lu LH, Moore MW: ErbB3 is required for normal cerebellar and cardiac development: a comparison with ErbB2-and heregulin-deficient mice. Development 1997, 124(24):4999-5011.

10. Britsch S, Goerich DE, Riethmacher D, Peirano RI, Rossner M, Nave KA, Birchmeier C, Wegner M: The transcription factor Sox10 is a key regulator of peripheral glial development. Genes Dev 2001, 15(1):66-78.

11. Lyons DA, Pogoda HM, Voas MG, Woods IG, Diamond B, Nix R, Arana N, Jacobs J, Talbot WS: erbb3 and erbb2 are essential for schwann cell migration and myelination in zebrafish. Curr Biol 2005, 15(6):513-524.

12. Honjo Y, Kniss J, Eisen JS: Neuregulin-mediated ErbB3 signaling is required for formation of zebrafish dorsal root ganglion neurons. Development 2008, 135(15):2615-2625.

13. Riethmacher D, Sonnenberg-Riethmacher E, Brinkmann V, Yamaai T, Lewin GR, Birchmeier C: Severe neuropathies in mice with targeted mutations in the ErbB3 receptor. Nature 1997, 389(6652):725-730.

14. Meyer D, Yamaai T, Garratt A, Riethmacher-Sonnenberg E, Kane D, Theill LE, Birchmeier C: Isoform-specific expression and function of neuregulin. Development 1997, 124(18):3575-3586.

15. Britsch S, Li L, Kirchhoff S, Theuring F, Brinkmann V, Birchmeier C, Riethmacher D: The ErbB2 and ErbB3 receptors and their ligand, neuregulin-1, are essential for development of the sympathetic nervous system. Genes Dev 1998, 12(12):1825-1836.

16. Gordon-Thomson C, Jones J, Mason RS, Moore GP: ErbB receptors mediate both migratory and proliferative activities in human melanocytes and melanoma cells. Melanoma Res 2005, 15(1):21-28.

17. Buac K, Xu M, Cronin J, Weeraratna AT, Hewitt SM, Pavan WJ: NRG1/ERBB3 signaling in melanocyte development and melanoma: inhibition of differentiation and promotion of proliferation. Pigment Cell Melanoma Res 2009, 22(6):773-784.

18. Budi EH, Patterson LB, Parichy DM: Embryonic requirements for ErbB signaling in neural crest development and adult pigment pattern formation. Development 2008, 135(15):2603-2614.

19. Baselga J, Swain SM: Novel anticancer targets: revisiting ERBB2 and discovering ERBB3. Nat Rev Cancer 2009, 9(7):463-475.

20. Kuhlbrodt K, Herbarth B, Sock E, Hermans-Borgmeyer I, Wegner M: Sox10, a novel transcriptional modulator in glial cells. J Neurosci 1998, 18(1):237-250. 
21. Southard-Smith EM, Kos L, Pavan WJ: Sox10 mutation disrupts neural crest development in Dom Hirschsprung mouse model. Nat Genet 1998, 18(1):60-64.

22. Herbarth B, Pingault V, Bondurand N, Kuhlbrodt K, Hermans-Borgmeyer I, Puliti A, Lemort N, Goossens M, Wegner M: Mutation of the Sry-related Sox10 gene in Dominant megacolon, a mouse model for human Hirschsprung disease. Proc Natl Acad Sci USA 1998, 95(9):5161-5165.

23. Pingault V, Bondurand N, Kuhlbrodt K, Goerich DE, Prehu MO, Puliti A, Herbarth B, Hermans-Borgmeyer I, Legius E, Matthijs G, et al: SOX10 mutations in patients with Waardenburg-Hirschsprung disease. Nat Genet 1998, 18(2):171-173.

24. Antonellis A, Huynh JL, Lee-Lin SQ, Vinton RM, Renaud G, Loftus SK, Elliot G, Wolfsberg TG, Green ED, McCallion AS, et al: Identification of neural crest and glial enhancers at the mouse Sox10 locus through transgenesis in zebrafish. PLOS Genet 2008, 4(9):e1000174.

25. Fisher S, Grice EA, Vinton RM, Bessling SL, McCallion AS: Conservation of RET regulatory function from human to zebrafish without sequence similarity. Science 2006, 312(5771):276-279.

26. Siepel A, Bejerano G, Pedersen JS, Hinrichs AS, Hou M, Rosenbloom K, Clawson H, Spieth J, Hillier LW, Richards S, et al: Evolutionarily conserved elements in vertebrate, insect, worm, and yeast genomes. Genome Res 2005, 15(8):1034-1050.

27. Heintzman ND, Stuart RK, Hon G, Fu Y, Ching CW, Hawkins RD, Barrera LO, Van Calcar S, Qu C, Ching KA, et al: Distinct and predictive chromatin signatures of transcriptional promoters and enhancers in the human genome. Nat Genet 2007, 39(3):311-318.

28. Creyghton MP, Cheng AW, Welstead GG, Kooistra T, Carey BW, Steine EJ, Hanna J, Lodato MA, Frampton GM, Sharp PA, et al: Histone H3K27ac separates active from poised enhancers and predicts developmental state. Proc Natl Acad Sci USA 2010, 107(50):21931-21936.

29. Rada-Iglesias A, Bajpai R, Swigut T, Brugmann SA, Flynn RA, Wysocka J: A unique chromatin signature uncovers early developmental enhancers in humans. Nature 2011, 470(7333):279-283.

30. Luscher B, Mitchell PJ, Williams T, Tjian R: Regulation of transcription factor AP-2 by the morphogen retinoic acid and by second messengers. Genes Dev 1989, 3(10):1507-1517.

31. Williams T, Admon A, Luscher B, Tjian R: Cloning and expression of AP-2, a cell-type-specific transcription factor that activates inducible enhancer elements. Genes Dev 1988, 2(12A):1557-1569.

32. Mitchell PJ, Timmons PM, Hebert JM, Rigby PW, Tjian R: Transcription factor AP-2 is expressed in neural crest cell lineages during mouse embryogenesis. Genes Dev 1991, 5(1):105-119.

33. Zhang J, Hagopian-Donaldson S, Serbedzija G, Elsemore J, PlehnDujowich D, McMahon AP, Flavell RA, Williams T: Neural tube, skeletal and body wall defects in mice lacking transcription factor AP-2. Nature 1996, 381(6579):238-241.

34. Schorle H, Meier P, Buchert M, Jaenisch R, Mitchell PJ: Transcription factor AP-2 essential for cranial closure and craniofacial development. Nature 1996, 381(6579):235-238.

35. Zhu CH, Huang Y, Oberley LW, Domann FE: A family of AP-2 proteins down-regulate manganese superoxide dismutase expression. J Biol Chem 2001, 276(17):14407-14413.

36. Zhu CH, Domann FE: Dominant negative interference of transcription factor AP-2 causes inhibition of ErbB-3 expression and suppresses malignant cell growth. Breast Cancer Res Treat 2002, 71(1):47-57.

37. Skinner A, Hurst HC: Transcriptional regulation of the c-erbB-3 gene in human breast carcinoma cell lines. Oncogene 1993, 8(12):3393-3401.

38. Inoue K, Khajavi M, Ohyama T, Hirabayashi S, Wilson J, Reggin JD, Mancias P, Butler IJ, Wilkinson MF, Wegner M, et al: Molecular mechanism for distinct neurological phenotypes conveyed by allelic truncating mutations. Nat Genet 2004, 36(4):361-369.

39. Fisher S, Grice EA, Vinton RM, Bessling SL, Urasaki A, Kawakami K, McCallion AS: Evaluating the biological relevance of putative enhancers using Tol2 transposon-mediated transgenesis in zebrafish. Nat Protoc 2006, 1(3):1297-1305.

40. Dutton JR, Antonellis A, Carney TJ, Rodrigues FS, Pavan WJ, Ward A, Kelsh RN: An evolutionarily conserved intronic region controls the spatiotemporal expression of the transcription factor Sox10. BMC Dev Biol 2008, 8:105.

41. Bamforth SD, Braganca J, Eloranta JJ, Murdoch JN, Marques FI, Kranc KR, Farza H, Henderson DJ, Hurst HC, Bhattacharya S: Cardiac malformations, adrenal agenesis, neural crest defects and exencephaly in mice lacking Cited2, a new Tfap2 co-activator. Nat Genet 2001, 29(4):469-474.

42. Dutton KA, Pauliny A, Lopes SS, Elworthy S, Carney TJ, Rauch J, Geisler R, Haffter P, Kelsh RN: Zebrafish colourless encodes sox10 and specifies non-ectomesenchymal neural crest fates. Development 2001, 128(21):4113-4125.

43. Dutton K, Dutton JR, Pauliny A, Kelsh RN: A morpholino phenocopy of the colourless mutant. Genesis 2001, 30(3):188-189.

44. Westerfield EM: The Zebrafish Book. A guide for the laboratory use of zebrafish (Danio rerio). University of Oregon Press; 2000.

45. Kimmel CB, Ballard WW, Kimmel SR, Ullmann B, Schilling TF: Stages of embryonic development of the zebrafish. Dev Dyn 1995, 203(3):253-310.

46. Cartharius K, Frech K, Grote K, Klocke B, Haltmeier M, Klingenhoff A, Frisch M, Bayerlein M, Werner T: MatInspector and beyond: promoter analysis based on transcription factor binding sites. Bioinformatics 2005, 21(13):2933-2942.

47. Antonellis A, Bennett WR, Menheniott TR, Prasad AB, Lee-Lin SQ, Green ED, Paisley D, Kelsh RN, Pavan WJ, Ward A: Deletion of long-range sequences at Sox10 compromises developmental expression in a mouse model of Waardenburg-Shah (WS4) syndrome. Hum Mol Genet 2006, 15(2):259-271.

48. Sviderskaya EV, Hill SP, Evans-Whipp TJ, Chin L, Orlow SJ, Easty DJ, Cheong SC, Beach D, DePinho RA, Bennett DC: p16(Ink4a) in melanocyte senescence and differentiation. J Nat/ Cancer Inst 2002, 94(6):446-454.

49. Toda K, Small JA, Goda S, Quarles RH: Biochemical and cellular properties of three immortalized Schwann cell lines expressing different levels of the myelin-associated glycoprotein. J Neurochem 1994, 63(5):1646-1657.

50. Lee TI, Johnstone SE, Young RA: Chromatin immunoprecipitation and microarray-based analysis of protein location. Nat Protoc 2006, 1(2):729-748.

51. Jones EA, Lopez-Anido C, Srinivasan R, Krueger C, Chang LW, Nagarajan R, Svaren J: Regulation of the PMP22 Gene through an Intronic Enhancer. J Neurosci 2011, 31(11):4242-4250.

\section{doi:10.1186/1471-213X-11-40}

Cite this article as: Prasad et al:: SOX10 directly modulates ERBB3 transcription via an intronic neural crest enhancer. BMC Developmental Biology 2011 11:40.

\section{Submit your next manuscript to BioMed Central and take full advantage of:}

- Convenient online submission

- Thorough peer review

- No space constraints or color figure charges

- Immediate publication on acceptance

- Inclusion in PubMed, CAS, Scopus and Google Scholar

- Research which is freely available for redistribution

Submit your manuscript at www.biomedcentral.com/submit
C Biomed Central 